\title{
RESEARCH ON ANTI-INTERFERENCE OF DOUBLE HOP WiRELESS POWERED COMMUNICATION NETWORKS BASED ON TIME REVERSAL
}

\author{
Wei Liu, Fang Wei Li, Jun Zhou Xiong and Ming Yue Wang \\ School of Communication and Information Engineering, Chongqing University \\ of Posts and Telecommunications, Chongqing, China
}

\begin{abstract}
To solve the problems of dual near and far in wireless powered communication network(WPCN) and the interference in the process of information transmission, a resource allocation method based on time inversal (TR) for WPCN is proposed. An optimization problem to maximize the minimum network throughput is constructed by jointly optimizing the transmission time of each phase of the network, the transmission power of the hybrid access point (HAP) and the transmission power of the relay. Since the constructed problem is non-convex, this paper converts the non-convex problem into an equivalent convex problem by introducing relaxation variables and auxiliary variables, and further divides the convex problem into two sub-problems to obtain the solution of the original problem. Finally, the simulation results show that the proposed resource allocation scheme can alleviate the dual distance and interference effectively, so as to obtain a higher total system throughput.
\end{abstract}

\section{KEYWORDS}

Wireless Powered Communication Network, Ttime Reversal, Jointly Optimizing

\section{INTRODUCTION}

In the Internet of Things era, different terminals need to be deployed due to different service types. As the number of terminals increases exponentially, massive sensor devices need to be deployed in the network to realize intelligent interconnection of everything. However, deploying a large number of sensor devices would face energy constraints, and regularly manually replacing or recharging batteries would be inconvenient, dangerous, or even impossible and costly[1]. The energy limitation problem has been alleviated by the development of energy harvesting technology, which uses the collected energy to charge network nodes [2], It is theoretically possible to provide equipment with permanent life Among many energy collection methods, RF signals are more suitable for small size and low power wireless node devices due to their predictable, controllable and stable nature [3], Therefore, RF energy collection is a feasible energy collection method. One of the most attractive research categories in the field of RF energy collection is the emerging Wireless Powered Communication Networks (WPCN), The network device can charge the battery by collecting energy from the surrounding environment or RF signals by dedicated energy transmitters [4], then uses the collected energy to fulfill its communication needs. Some scholars believe that WPCN is an integral part of the Internet of Things (IoT) [5], In this network, WPCN provide power to energy-constrained devices and are a 
new opportunity for the IoT. Therefore, WPCN system has attracted more and more attention [6].

During the downlink Wireless Enengy Trealation (WET) of WPCN, users who are farther away from Hybrid Access Point (HAP) get less energy than those who are closer, But in its uplink Wireless Inforemation Trealation (WIT) it must be transmitted with higher power, Call this phenomenon the dual near and far problem. In view of the dual near and far problem, some scholars have studied WET or WIT in the relay assisted WPCN, which is an effective way to solve the dual near and far problem and improve network performance. In [7], a multi-user relay WPCN is considered, which is based on the relay protocol of " charge than forward ", in which the single-antenna and half-duplex hybrid relay node first supplies power to the source node, and then the source and the relay can use the energy obtained from the hybrid relay node to upload the information to the destination. In [8] considers different WPCN scenarios composed of three nodes, in which multi-antenna relay not only WET the source node of a single antenna, but also cooperatively forward the information from the source node to the destination of the single antenna. In [9] considers a double-hop WPCN, in which the two-way communication between the user and the access point is assisted by a relay. The piecewise linear energy collection model of user and relay studies the problem of maximizing the total throughput of amplified and decoded relay mode, in which the energy transmitter is multiple antennas, the information access point and the user each have one antenna, and the relay has two antennas. Multi-antenna HAP is capable of downlink energy beamforming, which enables HAP to deliver more power to a specific user. In [9] addition to the multi-antenna system, the access point also adopts full-duplex communication, which improves the total throughput in full-duplex WPCN compared with halfduplex WPCN. Therefore, relay-assisted communication is an essential element in the IoT world. It expands coverage, improves availability, enhances reliability, increases network capacity, and reduces power consumption.

Another factor that affects throughput in WPCN is interference. In actual wireless communication scenarios, the total throughput of all users can be maximized as the number of terminals increases, but interference will increase [10]. The average interference of all HAP in multi-terminal WPCN is considered in [10], and the time allocation problem of achieving maximum and minimum throughput of all users in multi-cell WPCN is studied. A multi-antenna decoding and forwarding WPCN system is considered in [11]. The block descent method is used to jointly optimize transmit beamforming and self-interference cancellation in the process of combining and receiving. After repeated iterations, the optimization result is close to the optimal solution. In recent years, with the proposal and development of time reversal (TR) technology, research shows that TR can effectively alleviate the interference in the communication system. In [12] combines TR with multi-input and multi-output (MIMO) technology, and adopts the method of parameter research to quantitatively study the relationship between the performance of MIMO communication based on TR and the change of multi-path environment. The probability density function and cumulative distribution function of signal-to-noise ratio at the receiver in TR communication system are derived in [13]. Based on the derived probability density function, the traversal capacity, interrupt probability and bit error rate of binary phase shift keying are also obtained. The above research shows that in TR system, the inter-symbol interference decreases with the increase of the up-sampling factor, and the diversity gain increases with the increase of the number of paths. In [14] proposed that TR has tunneling effect in cloud wireless access network, which can effectively restrain the interference in the uplink transmission process and improve the system throughput. Broadband cooperative spectrum sensing based on FDMA technology is considered in [15]. TR generalized linear, TR maximum ratio combining and improved TR maximum ratio combining rules are proposed for decision fusion. The effectiveness of the proposed rules against inter-symbol interference (ISI) and inter-carrier interference(IUI) is tested according to the function of signal-to-interference-noise ratio(SINR). 
The research shows that compared with the traditional fusion rules, the performance of the fusion rules based on TR is greatly improved. Through the above research, we can know that TR can effectively alleviate the interference problem in the communication system, thus improving the performance of the system. TR makes use of the advantage of channel reciprocal focusing signal to suppress the transmission interference of uplink. Specifically, it uses the degree of freedom provided by the environment, that is, rich multipath, to use signature waveform design technology to combat interference. The basic idea of signature waveform design is to adjust the amplitude and phase of each tap of the signature waveform based on channel information so that the signal at the receiver can retain most of the useful signals and suppress interference as much as possible.

Through the review of the above work, we can see that the existing research work on WPCN is insufficient, as follows. First of all, in multi-terminal node networks, the nodes are randomly distributed, and it is the basic requirement of network application to ensure users' fair access to communication resources. Therefore, for WPCN with multiple communication nodes, the fairness among users can be ensured by calculating the minimum throughput maximization of terminal nodes. Secondly, resources are allocated only from the perspective of time. In the wireless power supply communication network, both time and power will affect the total throughput of the system, so it is necessary to jointly optimize to improve the network performance. Then, in the Internet of things, a large number of nodes, interference will also reduce network performance. Therefore, for the network with thousands of nodes, it is necessary to consider the combination of new technologies to reduce interference in the network and improve performance. Inspired by the above factors, in order to alleviate the problem of double distance to ensure user fairness and interference, this paper studies the resource allocation algorithm of dual-hop wireless communication network based on time reversal. The main work is as follows :

1. A multi-antenna double-hop WPCN model based on time reversal is proposed. Considering the constraints of time slot resources, HAP transmission power and relay node transmission power, the resource allocation problem of maximizing throughput is established.

2. Because the established optimization problem needs to jointly optimize time slots and power resources, resulting in the coupling of multiple variables, the proposed optimization problem is a non-convex optimization problem, so it is impossible to directly use the existing convex optimization tools to obtain the optimal solution. Therefore, this paper first transforms it into an equivalent convex problem by introducing relaxation variables and auxiliary variables, and then decomposes the optimal convex problem into two single-variable sub-problems, on this basis, the solution of the problem is obtained by using convex optimization theory.

3. Simulation results show that this algorithm can effectively alleviate the impact of double distance and interference problems. Compared with other schemes, it is proved that this algorithm can achieve better throughput performance.

The rest of this paper is organized as follows. A dual-hop WPCN model is introduced in section 2. Section 3 investigate the minimum throughput maximization problem, respectively. Section 4 evaluates the performance of the presented algorithms by conducting numerical simulations and section 5 concludes the paper.

\section{SySTEM MODEL}

As shown in Figure 1., this paper considers a dual-hop WPCN consisting of one HAP, one energy constrained relay node and $\mathrm{K}$ terminals. The HAP and relay nodes are equipped with $\mathrm{N}$ antennas, and the terminal is equipped with a single antenna. The transmission process of the 
whole system is completed by three processes: downlink WET, time reversal detection and uplink WIT. As shown in Figure 1. (a), the HAP transmits energy to the relay node and the terminal node in the downlink WET phase, and the relay node transmits detection signal detection channel state information to different terminals in the time reversal detection phase. The channel gains between the HAP to the relay node and to the terminal node $\mathrm{k}$ are defined as $H, h_{h k}$; The uplink WIT process is divided into two stages, as shown in Figure 1. (b), After the terminal node records the detected status information and performs TR operation, in the first stage of uplink WIT, the terminal node uses the energy stored in the downlink WET stage to send the information to the relay node, and in the second stage of uplink WIT, the relay centrally processes the received signal and forwards it to the HAP. Define the channel gain from the relay node to the terminal node $\mathrm{k}$ as $h_{r k}$.
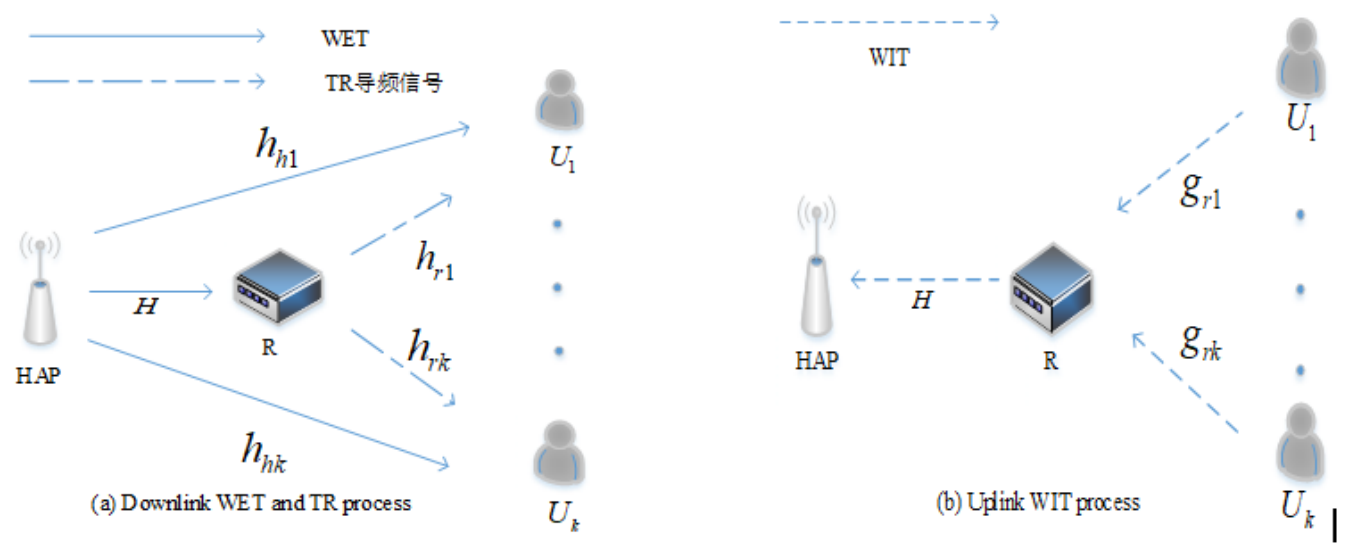

Figure 1. Dual hop WPCN system model

It is assumed that the relay and all terminal nodes are equipped with energy storage devices such as rechargeable batteries or super capacitors to store energy. The relay protocol of "charge than forward" is considered to coordinate energy and information transmission, in which HAP with constant energy supply firstly downlink transmits energy signals to charge relay and terminal nodes, and then relay and terminal nodes collect and store energy for uplink WIT. It is assumed that all channels between HAP, relay and terminal are reciprocal and experience slow, independent and flat Rayleigh fading, so that the channel gain remains unchanged within each transmission block $\mathrm{T}$, but varies independently between different transmission blocks. In the process of uplink WIT, the distance between the terminal and the HAP is very long or the signal is seriously attenuated, and there is no direct link between the HAP and the terminal, so relay assistance is needed to forward the information. Without losing generality, in the rest of this paper, assume that $\mathrm{T}=1$.

Considering the frame structure of a dual-hop WPCN with K terminals shown in Figure 2., one frame is divided into four time slots. In the first time duration $\tau_{0}$, HAP broadcasts an energy signal to the relay and all terminal nodes in order to provide energy for their upcoming uplink transmission tasks. In the second time duration $\tau_{t r}$, Firstly, the relay node sends detection signals to different terminals to obtain discrete channel impulse response; Then, each terminal records the discrete channel impulse response and inverts it in the time domain. After receiving the energy signal to supplement the battery, the terminal node uses the collected energy in the third time duration $\tau_{1}$ to convolute the information signal to be transmitted with the inverted discrete channel impulse response, and then sends it to the multi antenna relay node through 
SDMA. In the fourth time duration $\tau_{2}$, The relay node uses the energy stored in the downlink WET phase to forward the decoded information to the HAP.

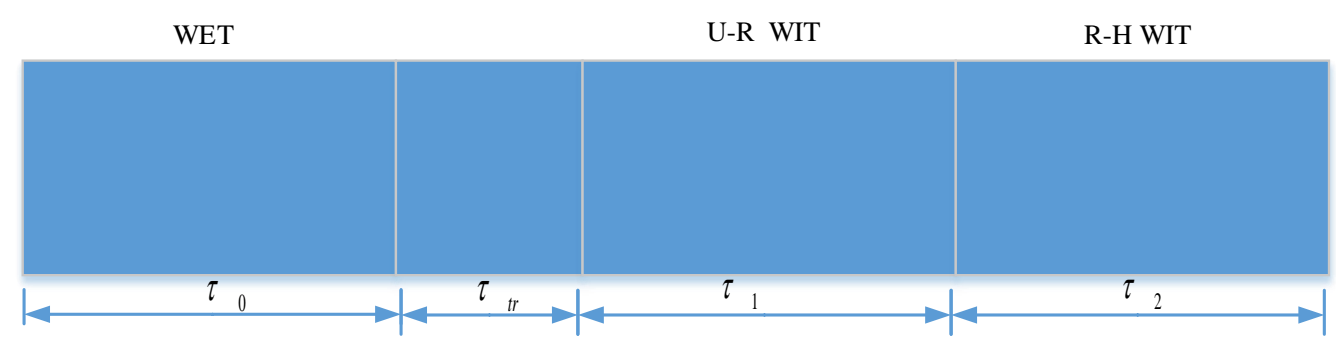

Figure 2. Frame structure of double hop WPCN network model

\subsection{Downlink WET Phase}

In the WET phase, HAP sends energy beams to the free battery multi antenna relay and all users at the same time. Let us defined $w_{l}$ as the energy beamforming weight transmitted by the $l$ antenna. The energy beamforming vector is defined as $w=\left(w_{1}, \ldots, w_{N}\right)^{T}$. The transmission power of antenna $l$ is $\left|w_{l}\right|^{2}$. The following limits are imposed on the transmit power of each antenna :

$$
\left|w_{l}\right|^{2} \leq P_{\max }
$$

$l=1, \ldots, N, \quad P_{\max }$ is the maximum transmit power of each antenna, || representative length.

The channel gain from HAP to battery free relay is expressed as $H \in \mathbf{C}^{N \times N}$, The channel gain from HAP to terminal $\mathrm{K}$ is defined as $h_{h k} \in \mathrm{C}^{N \times 1}, \quad 1 \leq k \leq K$. The signal received by the relay node and terminal is :

$$
\begin{aligned}
& y_{r}=H^{H} W+n_{r} \\
& y_{k}=h_{h k}^{H} W+n_{k}
\end{aligned}
$$

where $n_{r} \sim C N\left(\mu, \sigma_{r}^{2} I\right)$ is $N \times 1$ Additive White Gaussian Noise(AWGN), $n_{k} \sim N\left(0, \sigma_{k}{ }^{2}\right)$ is AWGN of terminal $U_{k}$, Superscript $H$ denotes conjugate transpose. The power obtained by the free battery relay in time slot $\tau_{0}$ can be expressed as :

$$
r_{r}=\left|y_{r}^{2}\right|=\left|H^{H} W\right|^{2}=\operatorname{tr}\left(W^{H} H H^{H} W\right)=\operatorname{tr}\left(H H^{H} W W^{H}\right)=\operatorname{tr}\left(G_{r} S\right)
$$

where $G_{r}=H H^{H} 、 S=\omega w^{H}$. The energy obtained by the free battery relay in the $\tau_{0}$ slot is :

$$
E_{H r}=\varepsilon \tau_{0} \operatorname{tr}\left(G_{r} S\right)
$$


Where $\varepsilon \in[0,1]$ is energy conversion efficiency, Similarly, the power obtained by the terminal in the $\tau_{0}$ slot can be expressed as :

$$
r_{k}=\left|y_{k}^{2}\right|=\left|h_{h k}^{H} W\right|^{2}=\operatorname{tr}\left(W^{H} h_{h k} h_{h k}^{H} W\right)=\operatorname{tr}\left(h_{h k} h_{h k}^{H} W W^{H}\right)=\operatorname{tr}\left(G_{k} S\right)
$$

Where $G_{k}=h_{h k} h_{h k}^{H}$

Similarly, the energy obtained by terminal $U_{k}$ in $\tau_{0}$ slot can be expressed as :

$$
E_{H k}=\varepsilon \tau_{0} \operatorname{tr}\left(G_{k} S\right)
$$

Then, the maximum transmission power of the relay node and terminal $U_{k}$ in the information transmission stage can be expressed as :

\subsection{TR Phase}

$$
P_{r}=\frac{\varepsilon \tau_{0} t r\left(G_{r} S\right)}{\tau_{2}}, \quad P_{k}=\frac{\varepsilon \tau_{0} t r\left(G_{k} S\right)}{\tau_{1}}
$$

In WPCN network, on the one hand, when multiple terminals transmit information uplink in SDMA mode, IUI will occur, resulting in system performance degradation. On the other hand, there are many terminals and their locations are randomly distributed. Pilot pollution will also affect the system performance. Rich multipath will produce inter symbol interference (ISI). Because the unique space-time focusing characteristic of TR technology can alleviate the channel delay and reduce the interference, this paper introduces TR technology and uses the unique space-time focusing characteristic of TR in multipath environment to counter IUI and ISI. Time focusing effect concentrates most of the useful signal energy of each symbol in a short time interval, which effectively suppresses ISI. Spatial focusing effect is to collect the signal energy at the expected position and reduce the leakage to other positions, resulting in the reduction of transmission power consumption and the co-channel interference to other positions.

The relay node and the terminal node receive the energy signal, the relay sends the detection signal to the terminal. The terminal inverts the discrete channel impulse response obtained by detection in the time domain to obtain the equivalent channel response, and normalizes it as a channel signature waveform. Each terminal transmits information through channels with different channel signatures, and effectively suppresses interference by adjusting the parameters of the channel signatures. The normalized channel signature waveform after TR processing is :

$$
g_{r k}(m)=\frac{h_{r k}^{*}(L-1-m)}{\sqrt{\sum_{m=0}^{L-1}\left|h_{r k}(m)\right|^{2}}}
$$

Where $*$ represents conjugation, $L$ is the number of multipath, $m=0, \ldots, L-1$. 


\subsection{Uplink WIT Phase}

In time slot $\tau_{1}$, all terminal nodes use the energy acquired in time slot $\tau_{0}$ to send information to relay nodes in SDMA, as shown in fig.1. According to transmission characteristics, the channel from the terminal to the relay node is regarded as a multi-access channel(MAC), so its capacity can be expressed by the following formula :

$$
C_{U-R}=\log _{2}\left|I+\sum_{k=1}^{K} \operatorname{SINR}\right|
$$

Where formula $I$ above is the identity matrix, SINR is :

$$
S I N R=\frac{P_{k} g_{r k} R_{k}^{0} g_{r k}^{H}}{P_{k} g_{r k} \hat{R}_{k} g_{r k}^{H}+\sum_{i \neq k} P_{i} g_{r k} R_{i} g_{r k}^{H}+\sigma^{2} I}
$$

where $p_{k}=\left[P_{1}, \ldots, P_{K}\right]^{T}$ is the uplink transmission power vector of the terminal, $R_{k}^{0}=A_{k}^{L} A_{k}^{(L) H}$ 、 $R_{i}=A_{i} A^{H}, A_{i}$ is the channel Topplitz matrix of the terminal node $i$ to relay node, $A_{k}^{L}$ represents the $L$ row vector of $A_{k}, \quad \hat{R}_{k}=A_{k} A_{k}^{H}-R_{k}^{0}$. In (11) $P_{k} g_{r k} \hat{R}_{k} g_{r k}^{H}$ and $\sum_{i \neq k} P_{i} g_{r k} R_{i} g_{r k}^{H}$ represents ISI and IUI. To simplify the calculation, it is assumed that the noise variances of different channels are the same.

In the time slot $\tau_{2}$, The relay node decodes the message received during $\tau_{1}$ and sends it to HAP through the MIMO channel. The singular value decomposition of the MIMO channel can be described as $H=U D V^{H}$, where $U$ and $V^{H}$ is the $N \times N$ unitary matrix. The diagonal elements of $D \in \mathbf{R}^{N \times N}$ are the eigen values of channel $H$, denoted by $\psi_{i}$. Its capacity is expressed as:

$$
C_{R-H}=\sum_{i=1}^{N} \log _{2}\left(1+\frac{P_{r i} \psi_{i}}{\sigma_{h}^{2}}\right)
$$

Where $\sigma_{h}^{2}$ denoes noise power, $N$ denotes the number of Gaussian channels, $P_{r i}$ is the transmission power of the $i$ th equivalent Gaussian channel. $P_{r i}$ can be calculated by waterfilling algorithm ${ }^{[16]}$. The calculation formula is : $P_{r i}=\left[\frac{1}{\gamma \times \operatorname{In} 2}-\frac{\sigma_{h}^{2}}{\psi_{i}}\right]^{+}$

Proof: please see Appendix A.

According to the above, the power has the following limitations $\sum_{i=1}^{N} P_{r i}=P_{r}$.

The throughput of the first hop and the second hop of the model proposed in this paper has been given, so the total throughput of the system can be expressed as: 


$$
R_{\text {sum }}=\min \left(\tau_{1} C_{U-R}, \tau_{2} C_{R-H}\right)
$$

The objective of this paper is to ensure the minimum rate of each terminal and maximize the total throughput of the system through joint optimization of time allocation and HAP downlink transmit energy beamforming matrix $S$. The minimum throughput maximization problem is shown as follows :

$$
\begin{aligned}
O P_{1}: & \max _{P_{r i}, P_{k}, \tau, w} R_{\text {sum }} \\
\text { s.t. } & C_{1}: \operatorname{tr}(S) \leq(K+1) P_{\max } \\
& C_{2}: \tau_{i} \geq 0, \quad \tau_{t r} \geq 0, \quad i=0,1,2 \\
& C_{3}: \tau_{0}+\tau_{t r}+\tau_{1}+\tau_{2}=1
\end{aligned}
$$

Where $C_{1}$ is maximum transmit Power constraint of HAP, $C_{2}$ and $C_{3}$ is time Duration constraint. This means that the sum of the duration of energy and information transmission must not exceed the length of the transmission block assumed to be $1 . \quad O P_{1}$ is non-convex, making it difficult to solve the problem, which will be solved below.

\section{Optimization Problem Solving}

In this part, we study and solve problem $O P_{1} . \quad O P_{1}$ is a non-convex problem of strongly coupled variables $\tau$ and $w$, which can not be solved in its original form. Therefore, in order to make the problem easy to deal with, this paper introduces new variables and uses the characteristics of sub-problems to solve. $O P_{1}$ is equivalent to the following questions:

$$
O P_{2}: \max _{w_{i}, \bar{R}, \tau} \bar{R}
$$

$$
\begin{aligned}
\text { s.t. } \quad C_{1}, \quad C_{2}, \quad C_{3} \\
C_{4}: \bar{R} \leq \tau_{1} \log _{2}\left|I+\sum_{k=1}^{K} \operatorname{SINR}\right| \\
C_{5}: \bar{R} \leq \tau_{2} \sum_{i=1}^{N} \log _{2}\left(1+\frac{P_{r i} \psi_{i}}{\sigma_{h}^{2}}\right)
\end{aligned}
$$

Where $\bar{R}$ is an introduced relaxation variable, The constraints of $C_{4}$ and $C_{5}$ are associated with the objective function expression. Obviously, $C_{U-R}$ and $C_{R-H}$ are joint concave functions in optimization variables, because they are the perspective of concave functions, and the perspective operator maintains concavity ${ }^{[17]}$. The objective function of $\mathrm{OP}_{2}$ and the constraints in $C_{1} 、 C_{2}$, and $C_{3}$ are affine functions. So for the new variables $w, \bar{R}$ and $\tau$ , problem $\mathrm{OP}_{2}$ is joint concave, and the problem can be effectively solved by a general convex optimization tool, such as CVX ${ }^{[18]}$. For the solution proposed above, because it contains matrix 
calculation, the computational complexity is very high. Therefore, this paper divides problem $\mathrm{OP}_{2}$ into Energy beamforming sub problem and time allocation sub problem to solve.

Given the time allocation $\tau$, the optimization method of energy beamforming is as follows:

In the proposed scheme, a beam splitting algorithm is used to separate the energy beams to provide energy to multiple nodes at the same time. Multi beam technology uses beamforming weight vector to realize Pareto optimality in received power domain. Pareto boundary $\left(R^{P F}\right)$ is defined as the set of all Pareto optimals in R. Expressed as follows:

$$
R^{P F}=\{x \in R \mid \text { not } r \in R \text { when } x \prec r\}
$$

where" $\prec "$ represents element inequality.

In this paper, a point on the Pareto boundary of $R$ is obtained by finding the maximum weighted sum of the received power vector in $R$. The optimization problem of the received power vector of the relay node and each terminal node is to maximize $\alpha^{T} x$ under the condition of $x \in R$, where $a=\left(\alpha_{0}, \ldots, \alpha_{K}\right)^{T}$ is the received power weight vector. The received power weight vector $a$ should satisfy $\alpha_{k} \geq 0$ and $1^{T} \alpha=1$. This optimization problem is equivalent to the following optimization problem:

$$
\begin{gathered}
\max : \alpha_{0}\left\|H^{H} W\right\|_{\infty}^{2}+\sum_{k=1}^{K} \alpha_{k}\left|h_{h k}^{H} W\right|^{2} \\
\text { s.t. }\left|w_{k}\right|^{2} \leq P_{\max } \quad k=0, \ldots, K
\end{gathered}
$$

It is difficult to directly solve the above optimization problem (16). Therefore, by relaxing the limit on the transmission power of each antenna, the total transmission power is limited. That is, $\quad \sum_{k=0}^{K}\left|w_{k}\right|^{2} \leq(K+1) P_{\max }$. Than, the optimization problem (16) is transformed into:

$$
\begin{gathered}
\max : \alpha_{0} \operatorname{tr}\left(G_{r} S\right)+\sum_{i=1}^{K} \alpha_{k} \operatorname{Ktr}\left(G_{k} S\right) \\
\text { s.t. } \operatorname{tr}(S) \leq(K+1) P_{\max }
\end{gathered}
$$

To solve the optimization problem (17), define:

$$
V(\alpha)=\alpha_{0} G_{r}+\sum_{i=1}^{K} \alpha_{k} G_{k}
$$

The eigenvalues of $V(\alpha)$ are decomposed into:

$$
V(\alpha)=U(\alpha)^{H} Z(\alpha) U(\alpha)
$$

Where $U(\alpha)$ is unitary matrix $U(\alpha)=\left(u_{1}(\alpha), \ldots, u_{N}(\alpha)\right), z(\alpha)$ is diagonal matrix $z(\alpha)=\operatorname{diag}\left(z_{1}(\alpha), \ldots, z_{N}(\alpha)\right)$. The diagonal elements in $z(\alpha)$ are arranged in descending order. $z_{1}(\alpha)$ is the principal eigenvalue. $u_{1}(\alpha)$ is the eigenvector corresponding to the principal 
eigenvalue $z_{1}(\alpha)$. Other eigenvectors are the same one-to-one correspondence. Therefore, the solution of (17) can be defined as $w^{o p}(\alpha)=\left(w_{1}^{o p}(\alpha), \ldots, w_{N}^{o p}(\alpha)\right)^{T}$, where :

$$
w^{o p}(\alpha)=\sqrt{(K+1) P_{\max }} u_{1}(\alpha)
$$

Since (20) is the solution of the relaxation optimization problem (17), the transmission power constraint of each antenna in (16) may not be satisfied and the maximum transmission power of each antenna is not fully utilized. Therefore, the transmission power of each antenna is adjusted in the following way to make the transmission power of each antenna $P_{\max }$ :

$$
w_{k}^{o p}(\alpha)=\frac{w_{k}^{o p}(\alpha)}{\left|w_{k}^{o p}(\alpha)\right|} \sqrt{P_{\max }}
$$

Where $W^{o p}(\alpha)=\left(w_{1}^{o p}(\alpha), \ldots, w_{N}^{o p}(\alpha)\right)^{T}$ is the optimal beamforming weight vector given $\alpha$. The optimal beamforming weight vector in (21) is the solution of (16).

For a given energy beamforming vector, the optimization problem of $\mathrm{OP}_{2}$ can be described as:

$$
\begin{aligned}
O P_{3} & : \max _{\bar{R}, \tau} \bar{R} \\
& \text { s.t. } \quad C_{2}, C_{3}, C_{4}, C_{5}
\end{aligned}
$$

It is worth noting that $\mathrm{OP}_{3}$ is a convex optimization problem. In this paper, we fix $\tau_{0}$ to find the optimal time allocation coefficient. In the case of fixed value $\tau_{0}$, the partial Lagrange of $\mathrm{OP}_{3}$ can be written as:

$$
\begin{aligned}
& L\left(\bar{R}, \tau_{0}, \tau_{t r}, \tau_{1}, \tau_{2} \lambda, \mu, v\right)=\bar{R}-\lambda\left(\tau_{0}+\tau_{t r}+\tau_{1}+\tau_{2}-1\right) \\
& -\mu\left(\bar{R}-\tau_{1} \log _{2}\left|I+\sum_{k=1}^{K} \operatorname{SINR}\right|\right)-v\left(\bar{R}-\tau_{2} \sum_{i=1}^{N} \log _{2}\left(1+\frac{P_{r i} \psi_{i}}{\sigma_{h}^{2}}\right)\right)
\end{aligned}
$$

Since $\mathrm{OP}_{3}$ is convex and satisfies Slater condition ${ }^{[17]}$, strong duality is established, and karush Kuhn Tucker (KKT) condition is a necessary and sufficient condition for the optimal solution. In order to obtain the optimal solution of the above dual function, it is necessary to maximize the Lagrange variable.

By considering the KKT condition related to $\bar{R}$, the following lemma is obtained. When finding the optimal throughput, the variable $\bar{R}$ and the dual variable must meet the optimal conditions. Lemma 1 : The optimal dual variables $\mu^{o p}$ and $v^{o p}$ must satisfy $0<\mu^{o p}<1$ and $0<v^{o p}<1$ Proof: Please see Appendix B

According to the result of lemma 1, the following proposition can be deduced: When the beamforming matrix is given, the throughput of the first hop from the terminal to the relay node and the throughput of the second hop from the relay node to the HAP must be equal. Namely: 


$$
\tau_{1}^{o p} C_{U-R}\left(\tau^{o p}\right)=\tau_{2}^{o p} C_{R-H}\left(\tau^{o p}\right)
$$

From lemma 1, dual variables must satisfy $0<\mu^{o p}<1$ and $0<v^{o p}<1$. From the KKT complementary relaxation condition in the proof process of lemma 1 , it can be easily proved that the optimal value should be satisfied $\bar{R}^{o p}=\tau_{1}^{o p} C_{U-R}\left(\tau^{o p}\right)=\tau_{2}^{o p} C_{R-H}\left(\tau^{o p}\right)$. Therefore, when the throughput of the first hop is equal to that of the second hop, the maximum total throughput can be obtained. And the optimal time allocation algorithm also needs the above conditions.

Lemma 2: When the energy beamforming matrix $S$ is given, and when $\tau_{1}$ and $\tau_{2}$ satisfy $\tau_{1}^{o p} C_{U-R}\left(\tau^{o p}\right)=\tau_{2}^{o p} C_{R-H}\left(\tau^{o p}\right)$, the throughput of the system is a concave function of $\tau_{0}$.

Proof: Please see Appendix C

When the beamforming energy matrix and the time $\tau_{0}$ of downlink transmission energy are given, $\tau_{1}$ and $\tau_{2}$ can be determined according to lemma 2 , When $\tau_{1}^{o p} C_{U-R}\left(\tau^{o p}\right)=\tau_{2}^{o p} C_{R-H}\left(\tau^{o p}\right)$ is satisfied, the throughput of the system is a concave function of $\tau_{0}$, and then the optimal solution is obtained according to the golden section algorithm.

Table 1. Time allocation optimization algorithm

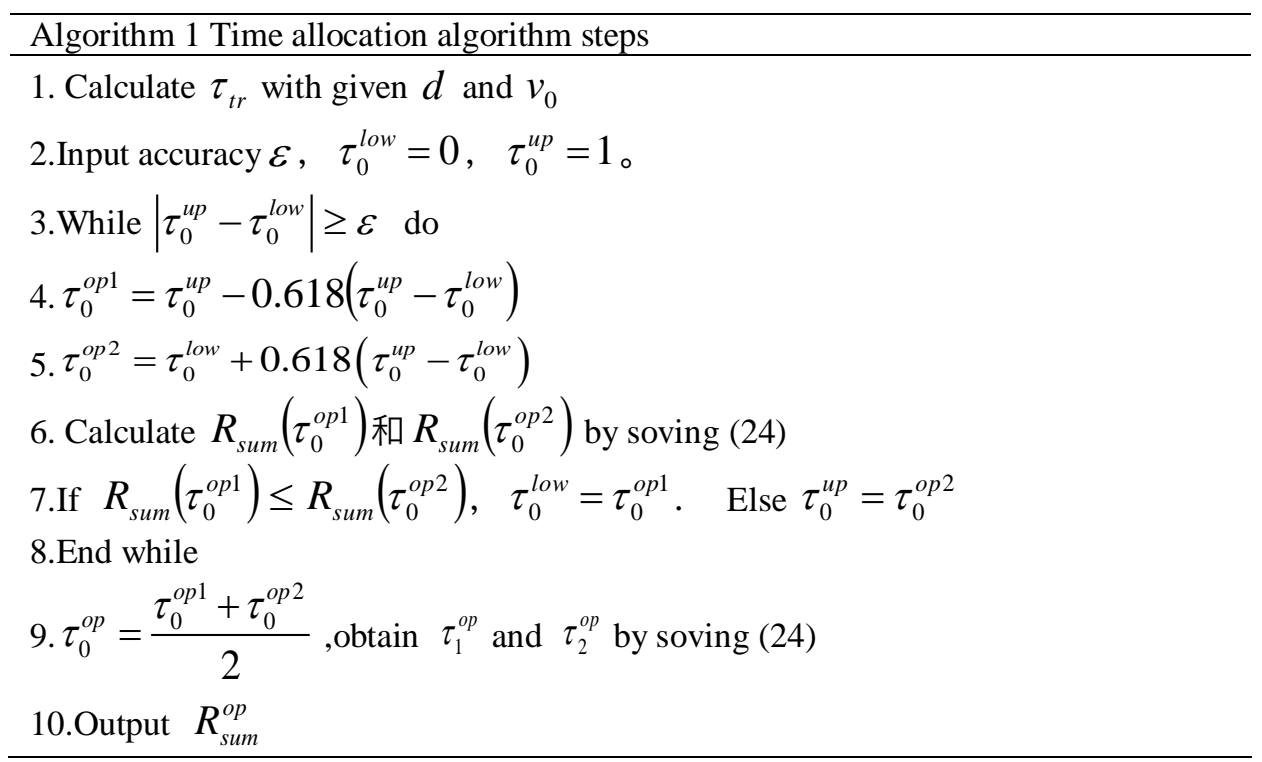

\section{Simulation ReSUlts}

For the multi antenna relay assisted WPCN in this paper, both HAP and free battery relay are equipped with $\mathrm{N}=6$ antennas. Assume the total bandwidth is $10 \mathrm{MHz}$, All channels experience quasi-static flat Rayleigh fading, and the channel power gain is modeled as a $10^{-3}\left(\frac{d_{i j}}{d_{0}}\right)^{-\alpha}$ , where $d_{i j}$ is the distance between relay node $\mathrm{i}$ and terminal $\mathrm{j}$, set $d_{0}$ as a reference distance of $1 \mathrm{~m}$ and $\alpha=3$ as the path loss factor. The maximum transmit power of HAP is set as 
$P_{\max }=40 \mathrm{dBm}$, The energy conversion efficiency is set as $\varepsilon=1$. This paper considers that in a two-dimensional Euclidean space with meter as unit, Place HAP in $(-2,0)$, The relay node is placed in the circle which center is $(0,0)$, The terminal node is uniformly placed in a circle which center is $(2,0)$ and a diameter of $1 \mathrm{~m}$. Unless otherwise stated, the channel model and simulation parameters are set the same. In this paper, the influence of this scheme on system performance under different conditions is analyzed and compared with the traditional scheme.

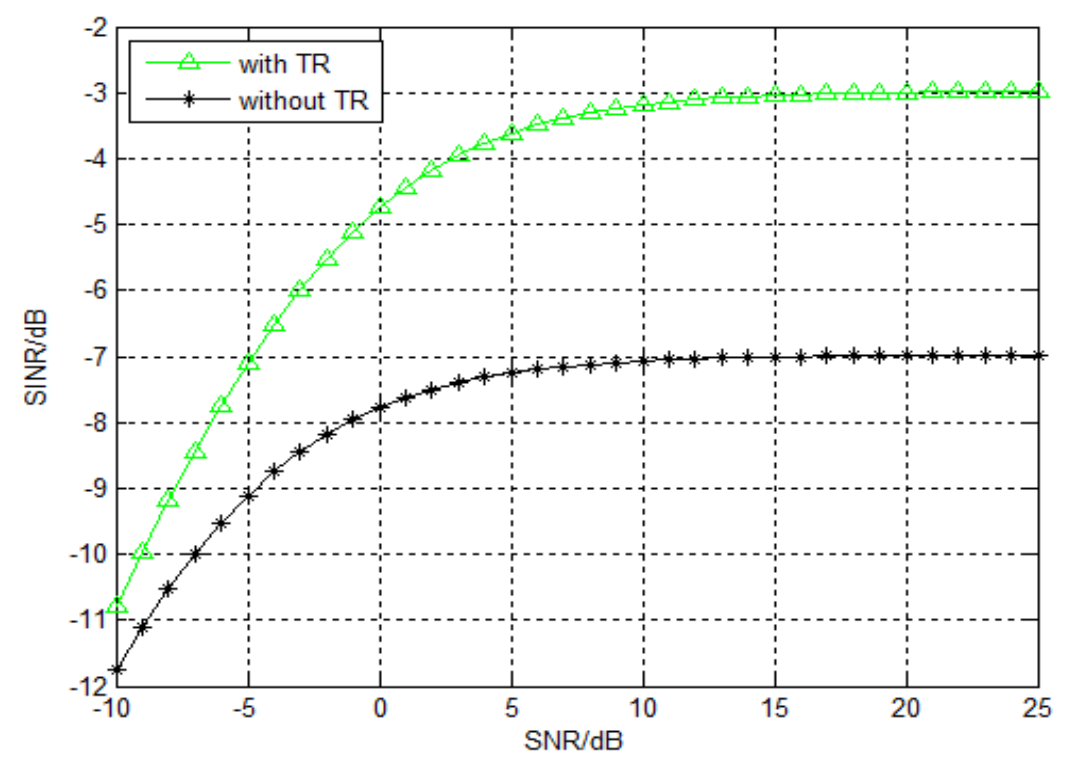

Figure 3. The relationship between SNR and SINR

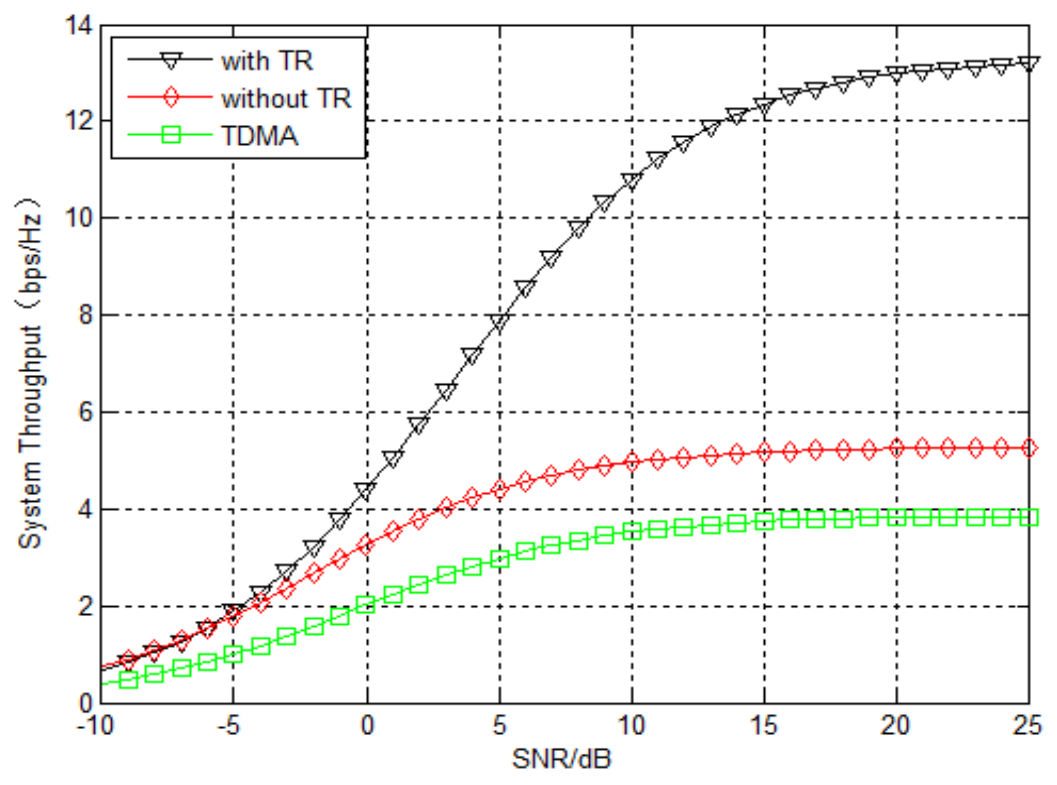

Figure 4. A comparison diagram of the throughput of different solutions 
Figure 3. shows the comparative analysis of SNR and SNIR with and without TR technology, it can be clearly seen that TR technology can effectively improve the SNIR. This is due to the fact that the space-time focusing of TR will focus at the same time and adaptively at the target point, resulting in higher signal amplitude and lower signal amplitude in other positions. Figure 4 shows the relationship between different SNR and the total throughput of the system under the same conditions. It can be seen from figure 4. that the proposed scheme can effectively improve the system throughput. Compared with the TDMA scheme with single antenna transmission, the system throughput of this scheme is significantly improved. In this paper, HAP and relay nodes are equipped with multi-antennas, and the downlink WET process adopts MIMO system multiuser beamforming technology, and the optimal beamforming power allocation scheme is obtained through research. Compared with the single antenna scheme, with the increase of the number of transmitting antennas, Higher directional gain can be obtained by beamforming in the direction of downlink energy concentrated transmission make up for the energy loss in the transmission process and improve the energy efficiency of WET. Therefore, the terminal node can get more energy for uplink WIT. And compared with the methods that do not use TR technology, TR technology effectively suppresses IUI and ISI in the uplink information transmission process, thus improving the system throughput and stability. In the scheme using TDMA, although there is no IUI, the uplink transmission consumes too much time, so the spectrum efficiency is low, and the relay is not used for secondary forwarding in the uplink WIT process, resulting in long transmission distance and large loss, which makes the system throughput relatively low. So, compared with the traditional time allocation scheme, the scheme proposed in this paper can effectively improve the total throughput of the system and make the system more stable. It can also be observed from Figure 4. that although the total throughput of the system increases with the increase of the SNR, when the SNR is too large, the throughput will reach balance state and will not change significantly with the increase of the SNR. Because with the improvement of SNR, although large capacity signals can be received more efficiently, the transmitted signals will be more interfered at the same time, so the system throughput reaches a balance state.

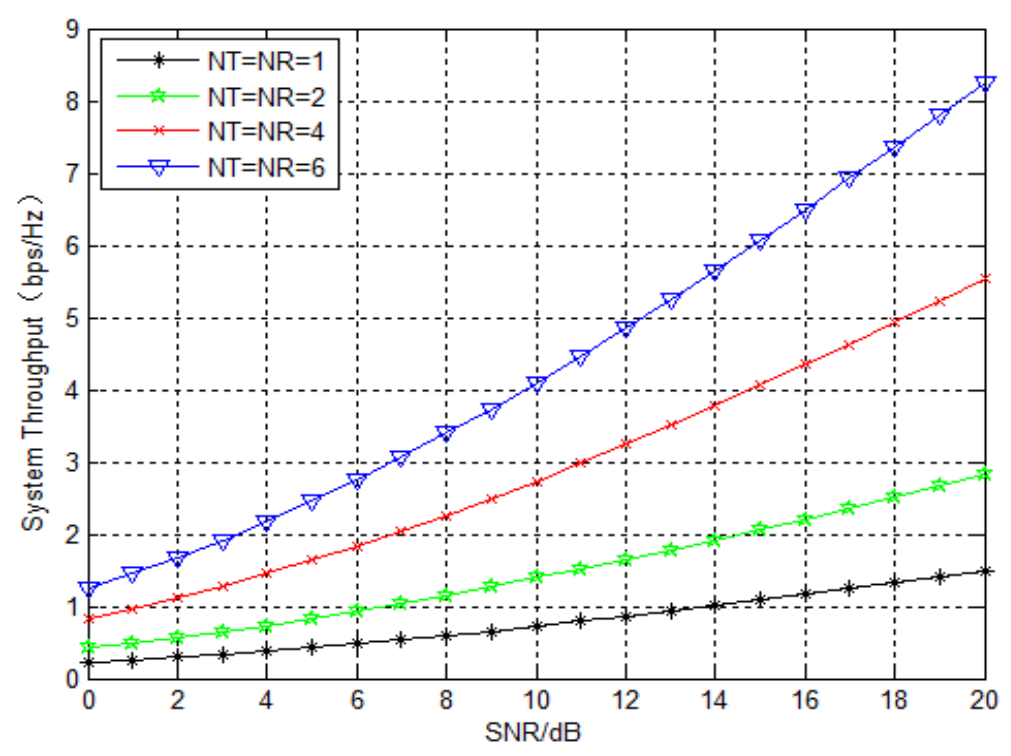

Figure 5. The relationship between total throughput and SNR

Figure 5. depicts the relationship between the total throughput of this paper scheme and the SNR under different antenna configurations of HAP and relay node. Monte Carlo simulation method 
is used in the simulation. Number of transmit receive antennas is $1 \times 1,2 \times 2 、 4 \times 4 、 6 \times 6$, The number of iterations of Monte Carlo simulation is 10000. As can be seen from Fig. 5, in the same case, the throughput increases with the increase of SNR. Compared with a single antenna, the greater the number of antennas, the greater the increase of throughput. It is proved that the application of multi-antenna in WPCN can effectively increase system throughput, and the effectiveness of the proposed scheme is also proved.

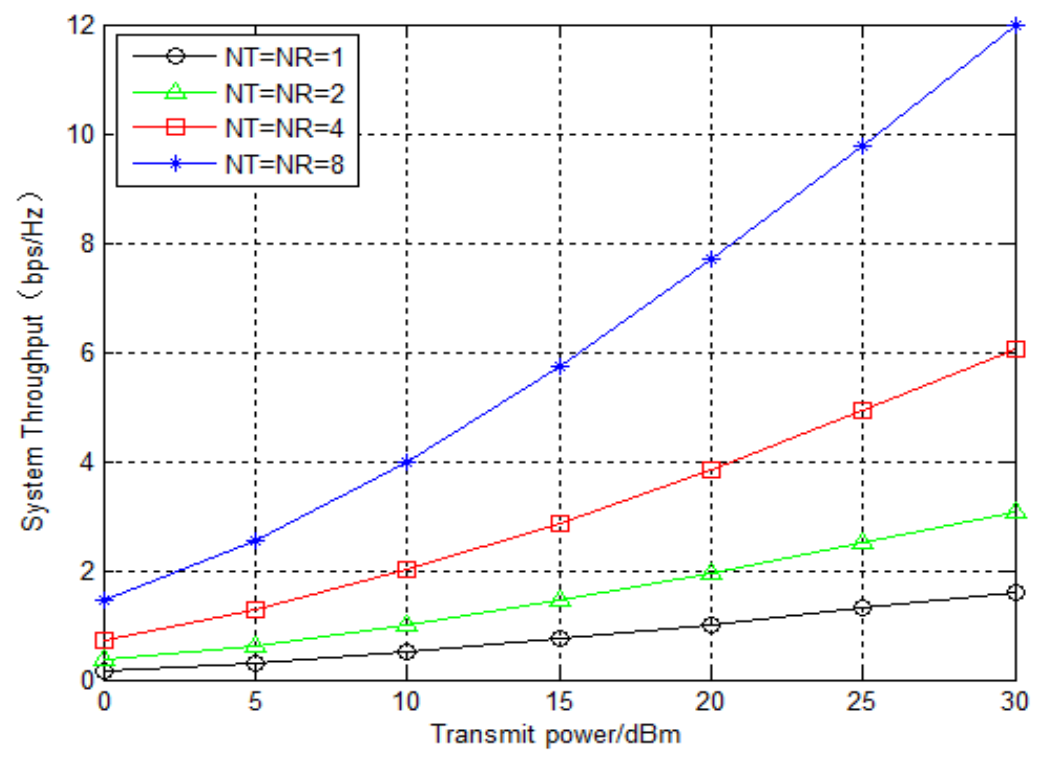

Figure 6. The relationship between total throughput and transmitted power

Figure 6. depicts the relationship between the total throughput of the system and the transmission power under different antenna configurations of HAP and relay nodes. For the scheme in this paper, it can be observed that the throughput increases with the increase of transmission power, and the multi-antenna scheme used in this paper has considerable gain. This is obvious, because by using higher transmission power, devices can collect more energy during WET, thus achieving higher throughput in WIT. It is clear in this figure that the multi-antenna scheme will always bring higher throughput than the single-antenna scheme. Because this paper applies relay to WPCN system, relay divides long-distance transmission into two hop transmission, which can reduce energy loss during transmission. After the first hop is added with TR technology, the space-time focusing characteristics of TR can effectively suppress the interference and information signal energy leakage during transmission, so the system performance can be improved.

\section{Conclusions}

For the WPCN, considering the double distance problem of the network itself and the interference will affect the system performance, By introducing relay and combining TR, this paper jointly optimizes the resource allocation problems such as the transmission time of each stage of the network, the transmission power of HAP and the transmission power of relay, in order to maximize the total throughput of the system. According to the model established by the proposed problem, the non-convex problem is transformed into an equivalent convex problem by introducing relaxation variables and auxiliary variables. Then the convex problem is divided into two sub-problems to obtain the suboptimal solution of the original problem. The simulation 
results show that the proposed scheme can suppress the interference and improve the system performance on the basis of effectively alleviating the double near far problem. The WPCN system has broad application prospects and can be used in wireless sensor networks. Future research can allocate and optimize resources for multi-antenna HAP and multi-user complex multi-cell environments and vehicle networking environments. The focusing intensity of time reversal technology depends on channel estimation. When there is an error in channel estimation, it will affect the focusing of time reversal. The scheme of this paper assumes that the channel state information is perfect, but in practice, the channel estimation will be affected by many factors in the wireless environment. Therefore, when there is an error in channel estimation, the performance of the system will be affected.

\section{ACKNOWLEDGEMENTS}

This work is supported by the Natural Science Foundation of China (NSFC) grant funded by the China government (61771084).

\section{APPENDIX A}

PROOF OF CALCULATION FORMULA

$$
\begin{aligned}
& \max : C_{R-H}=\sum_{i=1}^{N} \log _{2}\left(1+\frac{P_{r i} \psi_{i}}{\sigma_{h}^{2}}\right) \\
& \text { s.t. } \quad \sum_{i=1}^{N} P_{r i}=P_{r}
\end{aligned}
$$

The solution of the above problems can be solved by Lagrange multiplier method, and the corresponding Lagrange function can be written as :

$$
Z\left(\psi, P_{r i}\right)=\sum_{i=1}^{N} \log _{2}\left(1+\frac{P_{r i} \psi_{i}}{\sigma_{h}^{2}}\right)+\gamma\left(P_{r}-\sum_{i=1}^{N} P_{r i}\right)
$$

In the above formula, the partial derivative of $P_{r i}$ can be obtained :

$$
\begin{gathered}
\frac{\partial Z}{\partial P_{r i}}=\frac{1}{\operatorname{In} 2} \times \frac{\frac{\psi_{i}}{\sigma_{h}^{2}}}{1+\frac{P_{r i} \psi_{i}}{\sigma_{h}^{2}}}-\gamma=0 \\
P_{r i}=\frac{1}{\gamma \times \operatorname{In} 2}-\frac{\sigma_{h}^{2}}{\psi_{i}}
\end{gathered}
$$

Since the power cannot be negative, write the above formula as follows :

$$
P_{r i}=\left[\frac{1}{\gamma \times \operatorname{In} 2}-\frac{\sigma_{h}^{2}}{\psi_{i}}\right]^{+}
$$

Where $[a]^{+}=\max (a, 0)$ and $\gamma$ is constant. 


\section{APPENDIX B}

\section{PROOF OF LEMMA 1}

$$
\begin{gathered}
\frac{\partial L}{\partial \bar{R}}=1-\mu^{o p}-v^{o p}=0 \\
\mu^{o p}\left(\bar{R}^{o p}-\tau_{1}^{o p} \log _{2}\left|I+\sum_{k=1}^{K} \operatorname{SINR}\right|\right)=0 \\
v^{o p}\left(\bar{R}^{o p}-\tau_{2}^{o p} \sum_{i=1}^{N} \log _{2}\left(1+\frac{P_{r i}^{o p} \psi_{i}}{\sigma_{h}^{2}}\right)\right)=0
\end{gathered}
$$

Where $\mu^{o p}$ and $v^{o p}$ are the optimal dual variables of throughput constraint in $O P_{3}$. It is obvious from (31) that $v^{o p}=1-\mu^{o p}$ makes the Lagrangian dual function bounded, Using this result, $0<\mu^{o p}<1$ and $0<v^{o p}<1$ will be proved.

When $\mu^{o p}=0$ and $v^{o p}=1$.According to the KKT complementary relaxation condition in (33), it can be concluded that $\bar{R}^{o p}$ is the optimal solution that satisfies the throughput constraints in (15b) and is equal, i.e., $\bar{R}^{o p}=\tau_{2}^{o p} \sum_{i=1}^{N} \log _{2}\left(1+\frac{P_{r i}^{o p} \psi_{i}}{\sigma_{h}^{2}}\right)$. According to the KKT conditions in $\mu^{o p}=0$ and (32), $\quad \bar{R}^{o p}-\tau_{1}^{o p} \log _{2}\left|I+\sum_{k=1}^{K} \operatorname{SINR}\right|=0$ does not exist, because $\mu^{o p}=0$ means that the constraint in $C_{4}$ satisfies a strict inequality without loss of generality, i.e.,

$\bar{R}^{o p}<\tau_{1}^{o p} \log _{2}\left|I+\sum_{k=1}^{K} \operatorname{SINR}\right|$. Suppose there is $P^{1}$, so that

$\bar{R}^{1}\left(P^{1}\right)=\left(\tau_{1}^{o p} C_{U-R}\left(P^{1}\right)\right)^{1}=\left(\tau_{2}^{o p} C_{R-H}\left(P^{1}\right)\right)^{1} \quad$ and $\quad P^{o p}<P^{1}$, because $\tau_{2}^{o p} \sum_{i=1}^{N} \log _{2}\left(1+\frac{P_{r i}^{o p} \psi_{i}}{\sigma_{h}^{2}}\right)$ is an increasing function about $P$, it is get $\bar{R}^{o p}<\bar{R}^{1}$, which contradicts the assumption that $\bar{R}^{o p}$ is the optimal solution. Therefore, $\mu^{o p}$ cannot be 0 .

Than, The other case is $\mu^{o p}=1$, According to (31) know $v^{o p}=0$. By taking similar steps as before and considering the KKT conditions in (32) and (33), it is easy to get that $\bar{R}^{o p}$ is the optimal solution, so that $\bar{R}^{o p}=\tau_{1}^{o p} \log _{2}\left|I+\sum_{k=1}^{K} S I N R\right|$, without losing the generality of $\bar{R}^{o p}<\tau_{2}^{o p} \sum_{i=1}^{N} \log _{2}\left(1+\frac{P_{r i}^{o p} \psi_{i}}{\sigma_{h}^{2}}\right)$. Suppose there is $P^{2}, \quad$ so that $\bar{R}^{2}\left(P^{2}\right)=\left(\tau_{1}^{o p} C_{U-R}\left(P^{2}\right)\right)^{2}=\left(\tau_{2}^{o p} C_{R-H}\left(P^{2}\right)\right)^{2}$ and $P^{o p}<P^{2}$, because $\tau_{1} C_{U-R}$ is an increasing function about $P$, it is get $\bar{R}^{o p}\left(P^{o p}\right)<\left(\tau_{1}^{o p} C_{U-R}\left(P^{2}\right)\right)^{2}$, i.e. $\bar{R}^{o p}<\bar{R}^{2}$. Therefore, $\mu^{o p}$ cannot be 
1.If $\mu^{o p}>1$, then $v^{*}<0$, this violates the inequality constraint in the KKT condition where the dual variable is equal to or greater than zero. Therefore, it can be concluded that the optimal dual variable for the throughput constraint of $O P_{3}$ should satisfy $0<\mu^{o p}<1$ and $0<v^{o p}<1$.

\section{APPENDIX C}

\section{PROOF OF LEMMA 2}

$$
\begin{gathered}
R_{\text {sum }}\left(\tau_{0}\right)=\max _{E_{r}, E_{k}, \tau_{1}, \tau_{2}, \bar{R}} \bar{R} \\
\text { s.t. } C_{2}, C_{3} \\
C_{4}: E_{H r}=\varepsilon \tau_{0} \operatorname{tr}\left(G_{r} S\right) \\
C_{5}: E_{H k}=\varepsilon \tau_{0} \operatorname{tr}\left(G_{k} S\right) \\
R_{\text {sum }}\left(\tau_{0}\right)=\min \max _{E_{r}, E_{k}, \tau_{1}, \tau_{2}, \bar{R}} \bar{R}-a_{1} \tau_{0}-b_{1}\left(\tau_{t r}+\tau_{1}+\tau_{2}-1\right)-b_{2}\left(E_{H r}-\tau_{0} \varepsilon t r\left(G_{r} S\right)\right) \\
-b_{3}\left(E_{H k}-\tau_{0} \varepsilon \operatorname{tr}\left(G_{k} S\right)\right)
\end{gathered}
$$

Where $\bar{R}, \tau_{1}, \tau_{2}, S$ are the optimal solution. For arbitrary $\rho \in(0,1)$,

$$
\begin{aligned}
& R_{\text {sum }}\left(\rho x_{1}+(1-\rho) x_{2}\right)=\min _{a_{1 \geq 0}, b \geq 0} \\
& \bar{R}-a_{1}\left(\rho x_{1}+(1-\rho) x_{2}\right)-b_{1}\left(\tau_{t r}+\tau_{1}+\tau_{2}-1\right)-b_{2}\left(E_{H r}-\left(\rho x_{1}+(1-\rho) x_{2}\right) \operatorname{str}\left(G_{r} S\right)\right) \\
& -b_{3}\left(E_{H k}-\left(\rho x_{1}+(1-\rho) x_{2}\right) \varepsilon \operatorname{tr}\left(G_{k} S\right)\right) \\
& =\min _{a_{1} \geq 0, b \geq 0} \rho\left[\bar{R}-a_{1} x_{1}-b_{1}\left(\tau_{t r}+\tau_{1}+\tau_{2}-1\right)-b_{2}\left(E_{H \mathrm{r}}-x_{1} \operatorname{ctr}\left(G_{r} S\right)\right)-b_{3}\left(E_{H \mathrm{k}}-x_{1} \operatorname{ctr}\left(G_{k} S\right)\right)\right] \\
& +(1-\rho)\left[\bar{R}-a_{1} x_{2}-b_{1}\left(\tau_{t r}+\tau_{1}+\tau_{2}-1\right)-b_{2}\left(E_{H \mathrm{r}}-x_{2} \varepsilon \operatorname{tr}\left(G_{r} S\right)\right)-b_{3}\left(E_{H \mathrm{k}}-x_{2} \varepsilon \operatorname{tr}\left(G_{k} S\right)\right)\right](36)
\end{aligned}
$$

Since $\min _{x} f(x)+g(x) \geq \min _{x} f(x)+\min _{x} g(x)$ for $f(x)$ and $g(x) \in R$

So :

$$
\begin{aligned}
& R_{\text {sum }}\left(\rho x_{1}+(1-\rho) x_{2}\right) \geq \\
& \rho \min _{a_{1} \geq 0, b \geq 0}\left[\bar{R}-a_{1} x_{1}-b_{1}\left(\tau_{t r}+\tau_{1}+\tau_{2}-1\right)-b_{2}\left(E_{H \mathrm{r}}-x_{1} \varepsilon \operatorname{tr}\left(G_{r} S\right)\right)-b_{3}\left(E_{H \mathrm{k}}-x_{1} \varepsilon \operatorname{tr}\left(G_{k} S\right)\right)\right] \\
& +(1-\rho) \min _{a_{1} \geq 0, b \geq 0}\left[\bar{R}-a_{1} x_{2}-b_{1}\left(\tau_{t r}+\tau_{1}+\tau_{2}-1\right)-b_{2}\left(E_{H \mathrm{r}}-x_{2} \varepsilon \operatorname{tr}\left(G_{r} S\right)\right)-b_{3}\left(E_{H \mathrm{k}}-x_{2} \varepsilon \operatorname{tr}\left(G_{k} S\right)\right)\right] \\
& =\rho R_{\text {sum }}\left(x_{1}\right)+(1-\rho) R_{\text {sum }}\left(x_{2}\right)
\end{aligned}
$$

According to [17], $R_{\text {sum }}\left(\tau_{0}\right)$ is concave with $\tau_{0}$. 


\section{REFERENCES}

[1] KANG Xin, Ho C K, SUN Sumei. "Full-Duplex Wireless-Powered Communication Network with Energy Causality". IEEE Transactions on Wireless Communications, 2015, 14(10): 5539-5551.

[2] XU Jei, ZHANG Rui. "Throughput Optimal Policies for Energy Harvesting Wireless Transmitters with Non-Ideal Circuit Power”. IEEE Journal on Selected Areas in Communications, 2014, 32(2): 322-332.

[3] LU Xiao, WANG Ping, NIYATO D, et al. "Wireless Networks With RF Energy Harvesting: A Contemporary Survey". IEEE Communications Surveys \& Tutorials, 2015, 17(2): 757-789.

[4] MIKEKA C, ARAI H. "Design of a cellular energy-harvesting radio"// 2009 European Wireless Technology Conference. Rome, Italy. IEEE, 2009: 73-76

[5] RAMEZANI P, JAMALIPOUR A. "Toward the Evolution of Wireless Powered Communication Networks for the Future Internet of Things". IEEE Network, 2017, 31(6): 62-69.

[6] YANG Zhaohui, XU Wei, PAN Yijin, et al. "Optimal Fairness-Aware Time and Power Allocation in Wireless Powered Communication Networks". IEEE Transactions on Communications, 2018, 66(7): 3122-3135.

[7] LIU Mengyu, LIU Yuan. "Charge-Then-Forward: Wireless-Powered Communication for Multiuser Relay Networks". IEEE Transactions on Communications, 2018, 66(11): 5155-5167.

[8] HU Guojie, CAI Yueming, XU Kui, et al. "Opportunistic Energy Harvesting for Multi-AntennaRelay-Assisted Wireless Powered Communication Network". IEEE Communications Letters, 2019, 23(1): 148-151.

[9] RAMEZANI P, JAMALIPOUR A. "Two-Way Dual-Hop WPCN With A Practical Energy Harvesting Model". IEEE Transactions on Vehicular Technology, 2020, 69(7): 8013-8017.

[10] HE Chunlong, LIANG Jiaqian, QIAN Gongbin, et al. "Optimal Time Allocation in Multi-Cell Wireless Powered Communication Networks". IEEE Access, 2019, 7: 26519-26526.

[11] ZHENG Yali, HU Jie, YANG Kun. "Sum - Throughput Maximisation in Multi - Antenna aided FullDuplex WPCNs with Self-Interference"//2020 International Wireless Communications and Mobile Computing (IWCMC). Limassol, Cyprus. IEEE, 2020: 1240-1245.

[12] KIDA Y, DEGUCHI M, SHIMURA T. "The Effects of Interferences on Time Reversal MIMO: An Evaluation of Multipath and Co-Channel Interference"//2018 OCEANS - MTS/IEEE Kobe TechnoOceans (OTO). Kobe, Japan. IEEE, 2018: 1-5.

[13] LEI Weijia, YAO Li. "Performance Analysis of Time Reversal Communication Systems". IEEE Communications Letters, 2019, 23(4): 680-683.

[14] MA Hang, WANG Beibei, CHEN Yan, et al. "Waveforming Optimizations for Time-Reversal Cloud Radio Access Networks". IEEE Transactions on Communications, 2018, 66(1): 382-393.

[15] DEY I, CIUONZO D, ROSSI P S. "Wideband Collaborative Spectrum Sensing Using Massive MIMO Decision Fusion". IEEE Transactions on Wireless Communications, 2020, 19(8): 5246-5260.

[16] NIGUS H R, KIM K H, HWANG D. "Multi-antenna channel capacity enhancement in wireless communication"// 2015 Seventh International Conference on Ubiquitous and Future Networks. Sapporo, Japan. IEEE, 2015: 77-82.

[17] BOYD S, VANDENBERG L. "Convex optimization". Cambridge: Cambridge University Press, 2004: 61-256.

[18] GRANT M . CVX: MATLAB software for disciplined convex programming. http://cvxr.com/cvx, 2008. 


\section{AUTHORS}

Wei Liu Born in 1994, from Inner Mongolia, China. Now he is a master's student of Chongqing University of Posts and telecommunications. His main research interests are wireless power communication network and time reversal.

Fang Wei Li Born in 1960 in Chongqing, China. Professor and doctor of Chongqing University of Posts and telecommunications. His main research interests are electromagnetic field and electromagnetic wave, wireless network security, wireless transmission theory and technology.

Jun Zhou Xiong Born in 1985 in Hubei, China. Now he is a doctoral student of Chongqing University of Posts and telecommunications, and his main research direction is the key technology of wireless communication and physical layer security technology.

Ming Yue Wang Born in 1990 in Chongqing, China. Now her is a doctoral student of Chongqing University of Posts and telecommunications, and her main research direction is wireless transmission theory and technology.
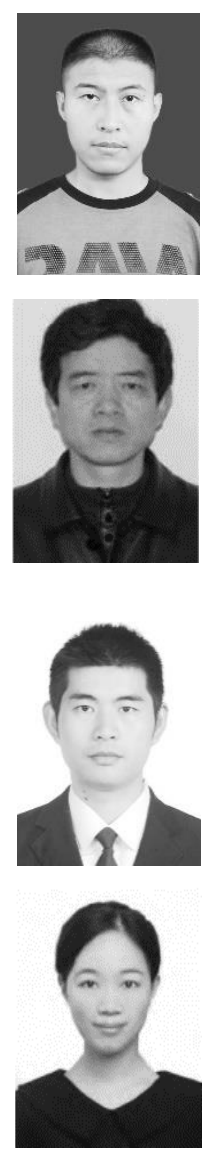

(C) 2022 By AIRCC Publishing Corporation. This article is published under the Creative Commons Attribution (CC BY) license. 